\title{
Approximate Distributions of the Residual Self-Interference Power in Multi-tap Full-Duplex Systems
}

\author{
Ayman T. Abusabah, Luis Irio, Rodolfo Oliveira, and Daniel B. da Costa
}

\begin{abstract}
In this letter, we investigate closed-form distributions to approximate the power of the residual Self-Interference (SI) due to: 1) uncanceled signals transmitted over multiple delaytaps, and 2) the presence of radio frequency and transceiver impairments, of an In-Band Full-duplex (IBFDX) wireless system. Starting with the distribution of the residual SI power for a single tap, we extend the analysis for multiple taps comparing two different solutions. The first one is based on the Welch-Satterthwaite (W-S) approximation, while the second is a moment-based approximation to an $\alpha-\mu$ distribution. When compared to empirical results obtained by simulation, our work shows that the distribution of the residual SI power can be accurately represented by the W-S approximation when the uncertainty level of the fading in the different taps is low. However, for higher levels of uncertainty we show that the $\alpha-\mu$ moment-based approximation is more accurate. A comparison between simulated and numerical results show the effectiveness of the proposed model.
\end{abstract}

Index Terms-In-Band Full-duplex Wireless Communications, Residual Self-interference, Stochastic Modeling.

\section{INTRODUCTION}

In In-Band Full-Duplex (IBFDX) communications, the nodes transmit and receive the signals simultaneously on the same frequency [1], exhibiting the potential of doubling the spectral efficiency. The residual self-interference (SI) is defined as the amount of remaining signal after the cancellation of the SI signal. The SI can be mitigated by a combination of passive and active methods [1]. Active methods can be adopted in both analog and digital domains [2]. The residual SI is mostly due to: (1) inaccurate SI channel estimates [3]; (2) radio frequency $(\mathrm{RF})$ and transceiver hardware impairments in the SI cancellation scheme [4].

Our work is mainly motivated by the importance SI cancellation in the digital domain, where the level of residual SI to be canceled is mainly caused by the wireless channel. Usually, the wireless channel and the SI are jointly estimated by exploiting known pilot symbols and the statistics of the residual SI [5]. However, the distribution of the residual SI is not symmetric, consequently, the statistics of the residual SI are not accurately characterized by only first and second moments, and the availability of high order statistics could enrich the joint estimation process. The distribution modeled

This work has received funding from the European Union's Horizon 2020 research and innovation programme under the Marie Skłodowska-Curie ETN TeamUp5G, grant agreement No. 813391, and by national funds through Fundação para a Ciência e Tecnologia (FCT), under the projects CoSHARE (PTDC/EEI-TEL/30709/2017) and UIDB/50008/2020.

A. T. Abusabah, L. Irio, and R. Oliveira are with the Instituto de Telecomunicações, 1049-001 Lisbon, Portugal. A. T. Abusabah and R. Oliveira are also with the Dep. de Engenharia Electrotécnica e de Computadores, Faculdade de Ciências e Tecnologia, Universidade Nova de Lisboa, 2829-516 Caparica, Portugal. D. B. da Costa is with the Dep. of Computer Engineering, Federal University of Ceará, 62010-560, Sobral, CE, Brazil. in the paper can thus be used to characterize the residual SI high order statistics that could enrich the estimation process in the digital domain.

In the recent literature, the statistical characterization of residual SI has received limited attention due to the difficulty of the mathematical modeling process. The amount of cancellation and the strength of the residual SI were computed in [3] for a single-tap delay channel. The similarity of the residual SI distribution with known distributions was analysed in [6], and a closed-form approximation was presented in [7]. While [3], [6] and [7] only consider a single-tap delay channel, our work advances the current research in the field by proposing closed-form approximations to the distribution of the residual SI power in multi-tap delay fading channels and assuming hardware impairments. The novelty of our work is the assumption of multi-tap delay fading channels and the comparison of two different methods to compute the distribution of the residual SI. To the best of the authors' knowledge, this is the first work characterizing the residual SI power for multiple fading taps.

This paper starts by considering that the multi-tap fading channel can be represented as a summation of independent non-identically distributed (i.n.i.d) Gamma random variables $(\mathrm{RVs})$. Due to the lack of a straightforward solution to represent the sum of i.n.i.d Gamma RVs, two different approximations are proposed to model the residual SI power: the Welch-Satterthwaite (W-S), and the $\alpha-\mu$. Both approximations are compared adopting low and high fading uncertainty levels associated to the taps and the occurrence of hardware impairments. We show that the $\alpha-\mu$ approximation outperforms the W-S approximation when the fading uncertainty levels are significantly high. On the other hand, the W-S approximation exhibits a high accuracy when the uncertainty levels are relatively low. In Section II, we present the architecture and mathematical formulation of multi-tap delay fading channel in IBFDX systems. In Section III, we derive the proposed model for the residual SI power. Numerical and simulated results are used to validate the model and presented in Section IV. Finally, Section V concludes the paper.

Notations: In this work, $f_{X}(\cdot)$ and $F_{X}(\cdot)$ represent the probability density function (PDF), and the cumulative distribution function $(\mathrm{CDF})$ of the $\mathrm{RV} X$, respectively. $\delta(\cdot)$ is used to represent Dirac's delta function. $\Gamma(\cdot)$ represents the complete Gamma function. $K_{x}(\cdot)$ represents the modified Bessel function of the second kind and order $x$. A Gaussian distribution with mean $\mu$ and variance $\sigma^{2}$ is denoted by $\mathcal{N}\left(\mu, \sigma^{2}\right)$. Gamma $(k, \theta)$ represents a Gamma distribution with shape $(k)$ and a scale $(\theta)$ parameters. $\operatorname{Nakagami}(m, \lambda)$ denotes the Nakagami distribution with a shape $(m)$ and spread $(\lambda)$ parameters. The notation $\chi_{k}^{2}$ denotes a chi-squared 
distribution with $k$ degree of freedom. $E[X]$ and $\operatorname{Var}[X]$ are the expectation and variance of the RV $X$, respectively. A RV $Y$ is a realization of a time-varying signal, $y(t)$.

\section{SYSTEM MODEL}

\section{A. In-Band Full Duplex Canceller}

In this work a post-mixer active analog canceler is adopted, where the canceling signal is generated by processing the SI signal after the RF upconversion stage, as considered in [3] and [7]. The block diagram of the analog canceler is depicted in Fig. 1. The main goal is to characterize the residual interference, $y_{\text {res }}(t)$, after the analog cancellation, so it can be used in the digital domain.

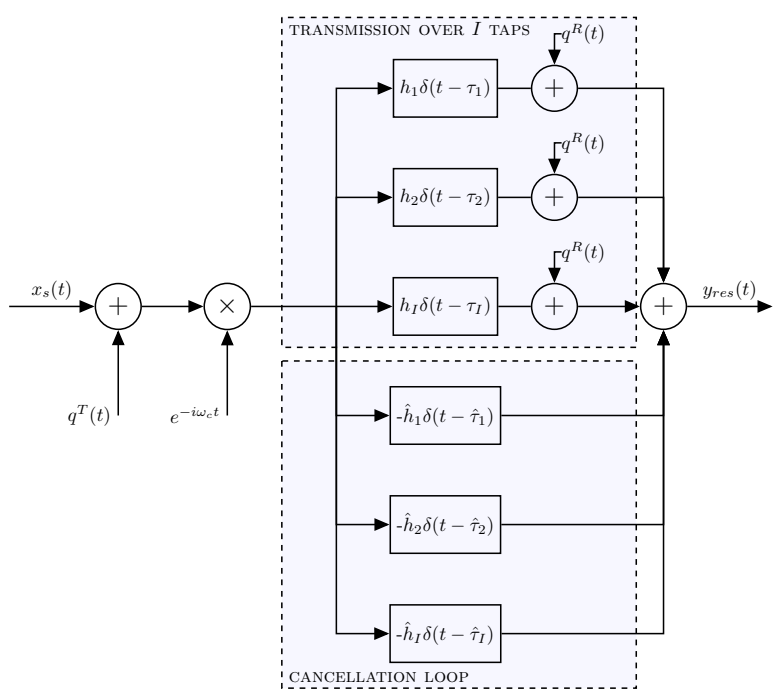

Fig. 1. Multi-tap analog post-mixer canceller.

The cancellation loop admits $I$ multi-tap delay fading channels. The active analog canceler reduces the SI at the angular carrier frequency $\omega_{c}=2 \pi f_{c}$. Multiple shifted versions of the SI signal, $x_{s}(t)$, with different amplitudes are expected at the receiver side. The $i$-th tap is characterized by the delay and the channel gain, $\tau_{i}$ and $h_{i}$, respectively. We consider RF and hardware impairments, $q^{T}(t)$ and $q^{R}(t)$, at the transmitter and receiver chains, respectively. The combined influence of the impairments has been studied in [8] and used in many IBFDX works [9]. In particular, the $x_{s}(t)$ signal is distorted by $q^{T}(t)$, while the receiver chains are subjected to a $q^{R}(t)$ distortion, as represented in Fig. 1. With the aim of minimizing the residual SI signal, $y_{\text {res }}(t)$, the estimated delay $\hat{\tau}_{i}$ and estimated gain, $\hat{h}_{i}$, related to the $i$-th tap, have to be injected in the cancellation loop. Note that, the $\hat{h}_{i}$ has to capture the impairments contribution in order to be removed at the cancellation stage. Thus, $\hat{h}_{i}=\epsilon_{i}\left(h_{i}+\frac{q^{R}(t)}{x_{s}(t)+q^{T}(t)}\right)$, where $\left(1-\epsilon_{i}\right)$ is the gain estimation error of the $i$-th tap, $\epsilon_{i} \geq 0$. The phase estimation error of the $i$-th tap is given by $\phi_{i}=\omega_{c}\left(\tau_{i}-\hat{\tau}_{i}\right)$. The estimation of the parameters $\hat{\tau}_{i}$ and $\hat{h}_{i}$ can be done using different methods already available in the literature, such as the one described in [10].

The residual SI signal, $y_{\text {res }}(t)$, not canceled by the analog cancelation process, is discretized over time. The proposed analysis uses the discretized samples of $y_{r e s}(t)$ as a starting point to derive its distribution. In this way, we characterize the distribution of the residual SI at the output of the analog cancelation scheme, being available for the digital domain signal processing stage.

\section{B. Residual Self-Interference Power}

According to Fig. 1, the residual SI, $y_{\text {res }}(t)$, can be expressed mathematically as follows

$$
\begin{aligned}
y_{r e s}(t)= & \sum_{i=1}^{I}\left(x_{s}\left(t-\tau_{i}\right)+q^{T}\left(t-\tau_{i}\right)\right) e^{j \omega_{c}\left(t-\tau_{i}\right)} h_{i}+q^{R}(t) \\
& -\left(x_{s}\left(t-\hat{\tau}_{i}\right)+q^{T}\left(t-\hat{\tau}_{i}\right)\right) e^{j \omega_{c}\left(t-\hat{\tau}_{i}\right)} \hat{h}_{i} .
\end{aligned}
$$

Channel gains are assumed to be independent and complex, i.e., $h_{i}=h_{r_{i}}+j h_{j_{i}}$. We also assume that $x_{s}(t)$ and $q^{T, R}(t)$ are independent complex signals given by $x_{s}(t)=x_{r}(t)+j x_{j}(t)$ and $q^{T, R}(t)=q_{r}^{T, R}(t)+j q_{j}^{T, R}(t)$, respectively. Considering $x_{s}\left(t-\tau_{i}\right) \approx x_{s}\left(t-\hat{\tau}_{i}\right), q^{T}\left(t-\tau_{i}\right) \approx q^{T}\left(t-\hat{\tau}_{i}\right)$ [7], and using Lemma 1 in [8], (1) can be represented as follows

$$
y_{\text {res }}(t)=\left(x_{s}(t)+q(t)\right) \sum_{i=1}^{I} h_{i} c_{i},
$$

where $q(t)$ is the aggregate distortion noise capturing contributions from hardware impairments at both the transmitter and the receiver, and $c_{i}=\left(e^{j \omega_{c}\left(t-\tau_{i}\right)}-\epsilon_{i} e^{j \omega_{c}\left(t-\hat{\tau}_{i}\right)}\right)$. Thus, the residual SI power can be expressed as

$$
P_{y_{\text {res }}}=S H \text {. }
$$

The RV $S=S_{r}^{2}+S_{j}^{2}$ represents the power of the SI signal, and the power of the aggregate hardware impairments, where $S_{r}=\left(X_{r}+Q_{r}\right)$ and $S_{j}=\left(X_{j}+Q_{j}\right)$. Moreover, the RV $H=\sum_{i=1}^{I}\left(H_{r_{i}}^{2}+H_{j_{i}}^{2}\right) C_{i}$ represents the power of fading taps and estimation errors. The constant $C_{i}=\left(1+\epsilon_{i}^{2}-2 \epsilon_{i} \cos \left(\phi_{i}\right)\right)=\left(\left(\Re\left(c_{i}\right)\right)^{2}+\left(\Im\left(c_{i}\right)\right)^{2}\right)$ represents the power of $c_{i}$.

\section{Self-Interference POWER Distribution}

This section considers the required steps to derive the distribution of the residual SI power, denoted by $P_{y_{r e s}}$.

\section{A. Characterization of $S$}

Regarding the transmitted signal, $x_{s}(t)$, we assume that it is a circularly-symmetric complex signal, with $\left\{X_{r}, X_{j}\right\} \sim$ $\mathcal{N}\left(0, \sigma_{x}^{2}\right)$. The circularly-symmetric complex distribution is considered because it can effectively represent Orthogonal Frequency-Division Multiplexing systems with a high number of carriers. The transmitter and receiver impairments are defined as $\left\{Q_{r}^{T}, Q_{j}^{T}\right\} \sim \mathcal{N}\left(0, \kappa_{T}^{2}\right)$ and $\left\{Q_{r}^{R}, Q_{j}^{R}\right\} \sim$ $\mathcal{N}\left(0, \kappa_{R}^{2}|h|^{2}\right)$, respectively, with design parameters $\kappa_{T}, \kappa_{R} \geq$ 0 , [8], [9]. Consequently, the aggregate effect of both transmitter and receiver impairments, $q(t)$, can be then characterized by $\left\{Q_{r}, Q_{j}\right\} \sim \mathcal{N}\left(0, \kappa^{2}\right)$ where $\kappa=\sqrt{\kappa_{T}^{2}+\kappa_{R}^{2}}$ represents the aggregate level of impairments, ([8], Lemma 1). Because the sum of two independent normally distributed RVs is 
another normal distribution, then $\left\{S_{r}, S_{j}\right\} \sim \mathcal{N}\left(0, \sigma_{s}^{2}\right)$, where $\sigma_{s}^{2}=\sigma_{x}^{2}+\kappa^{2}$. Consequently, the RVs $S_{r}^{2}$ and $S_{j}^{2}$ follow a scaled Chi-squared distribution with $k=1$ degrees of freedom denoted by $\chi_{1}^{2}$ and may be written as follows

$$
\left\{S_{r}^{2}, S_{j}^{2}\right\} \sim \sigma_{s}^{2} \chi_{1}^{2} .
$$

By definition, if $Q \sim \chi_{k}^{2}$ and $v$ is a positive constant, then $v Q \sim \operatorname{Gamma}(k / 2,2 v)$. Consequently,

$$
\left\{S_{r}^{2}, S_{j}^{2}\right\} \sim \operatorname{Gamma}\left(1 / 2,2 \sigma_{s}^{2}\right) .
$$

Finally, the sum of two Gamma RVs, holding the same scale parameter, results another Gamma distribution, i.e., $\operatorname{Gamma}\left(k_{1}, \theta\right)+\operatorname{Gamma}\left(k_{2}, \theta\right)=\operatorname{Gamma}\left(k_{1}+k_{2}, \theta\right)$, thus,

$$
S=S_{r}^{2}+S_{j}^{2} \sim \operatorname{Gamma}\left(1,2 \sigma_{s}^{2}\right) .
$$

\section{B. Characterization of $H$}

The RV $H$ includes $I$ independent Rician fading taps along with estimation errors. To characterize its distribution, we first consider the distribution of a single tap. The Rician fading tap is parameterized through $K$ and $\Omega . K$ represents the quotient between the power in the LoS component and the power in the other non-LoS components. $\Omega$ represents the total power from both components. Then, the received signal amplitude of the $i$-th tap is Rician distributed with parameters $\mu_{h_{i}}^{2}=\frac{K_{i} \Omega_{i}}{1+K_{i}}$ and $\sigma_{h_{i}}^{2}=\frac{\Omega_{i}}{2\left(1+K_{i}\right)} . K_{d B_{i}}=10 \log _{10}\left(K_{i}\right)$ is the decibels representation of $K_{i}$.

If the $i$-th tap is Rician, then, $H_{r_{i}} \sim \mathcal{N}\left(\mu_{h_{i}} \cos \left(\vartheta_{i}\right), \sigma_{h_{i}}^{2}\right)$ and $H_{j_{i}} \sim \mathcal{N}\left(\mu_{h_{i}} \sin \left(\vartheta_{i}\right), \sigma_{h_{i}}^{2}\right)$. Consequently, the term $\left(1 / \sigma_{h_{i}}^{2}\right)\left(H_{r_{i}}^{2}+H_{j_{i}}^{2}\right)$ follows a non-central Chi-squared distribution with $k=2$ degrees of freedom and non-centrality parameter $\mu_{h_{i}}^{2} / \sigma_{h_{i}}^{2}$. Using the method of moments, we end up with a simplified Gamma approximation to approximate the shape and scale parameters, $k_{h_{i}}$ and $\theta_{h_{i}}$, respectively, given by

$$
k_{h_{i}}=\frac{\left(\mu_{h_{i}}^{2}+2 \sigma_{h_{i}}^{2}\right)^{2}}{4 \sigma_{h_{i}}^{2}\left(\mu_{h_{i}}^{2}+\sigma_{h_{i}}^{2}\right)}, \theta_{h_{i}}=\frac{4\left(\mu_{h_{i}}^{2}+\sigma_{h_{i}}^{2}\right)}{\left(\mu_{h_{i}}^{2}+2 \sigma_{h_{i}}^{2}\right)} .
$$

Since $C_{i}$ is a constant, the term $\left(H_{r_{i}}^{2}+H_{j_{i}}^{2}\right) C_{i}$ can be written as follows

$$
H_{i}=\left(H_{r_{i}}^{2}+H_{j_{i}}^{2}\right) C_{i} \sim \operatorname{Gamma}\left(k_{i}, \theta_{i}\right),
$$

where $k_{i}=k_{h_{i}}$ and $\theta_{i}=\theta_{h_{i}} \sigma_{h_{i}}^{2} C_{i}$. Therefore, the RV $H$ is merely a sum of i.n.i.d Gamma RVs and may be written as follows

$$
H=\sum_{i=1}^{I} H_{i}
$$

\section{Welch-Satterthwaite Approximation}

Due to intractability of closed-form solutions for the sum of i.n.i.d Gamma RVs, we use the W-S method for the approximation of $H$. The original W-S approximation was for linear combinations of independent Chi-square RVs. However, their basic idea easily extended to the sum of i.n.i.d Gamma RVs [11], [12].
Let $\left\{H_{i}\right\}_{i=1}^{I}$ be i.n.i.d Gamma RVs with $H_{i} \sim$ $\operatorname{Gamma}\left(k_{i}, \theta_{i}\right)$, and $H=H_{1}+\cdots+H_{I}$ be their sum, then

$$
H \sim \operatorname{Gamma}\left(k_{e q}, \theta_{e q}\right),
$$

where $k_{e q}$ and $\theta_{e q}$ are the equivalent shape and scale parameters, respectively, and given by

$$
k_{e q}=\frac{\left(k_{1} \theta_{1}+\ldots+k_{I} \theta_{I}\right)^{2}}{k_{1} \theta_{1}^{2}+\ldots+k_{I} \theta_{I}^{2}}, \theta_{e q}=\frac{k_{1} \theta_{1}^{2}+\ldots+k_{I} \theta_{I}^{2}}{k_{1} \theta_{1}+\ldots+k_{I} \theta_{I}} .
$$

Substituting (7) in (11), we obtain

$$
k_{e q}=\frac{\left(\sum_{i=1}^{I} k_{h_{i}} \theta_{h_{i}} \sigma_{h_{i}}^{2} C_{i}\right)^{2}}{\sum_{i=1}^{I} k_{h_{i}}\left(\theta_{h_{i}} \sigma_{h_{i}}^{2} C_{i}\right)^{2}}, \quad \theta_{e q}=\frac{\sum_{i=1}^{I} k_{h_{i}}\left(\theta_{h_{i}} \sigma_{h_{i}}^{2} C_{i}\right)^{2}}{\sum_{i=1}^{I} k_{h_{i}}\left(\theta_{h_{i}} \sigma_{h_{i}}^{2} C_{i}\right)} .
$$

By definition, $S$ and $H$ are independent. Thus, the PDF of $P_{y_{\text {res }}}$ can be found by computing the product density function as follows

$$
f_{P_{y_{r e s}}}(z)=\int_{-\infty}^{\infty} f_{S}(s) f_{H}(z / s) \frac{1}{|s|} d s .
$$

The integral in (13) is solved replacing $f_{S}(s)$ and $f_{H}(z / s)$ by (6) and (10), respectively, obtaining the PDF of the residual SI power as follows [7]

$$
\begin{aligned}
f_{P_{y_{r e s}}}(z) \approx & \frac{2^{\frac{1-k_{e q}}{2}} \sigma_{s}^{-k_{e q}-1} \theta_{e q}^{-k_{e q}}}{\Gamma\left(k_{e q}\right)} \\
& \times\left(\theta_{e q} / z\right)^{\frac{k_{e q}-1}{2}} z^{k_{e q}-1} K_{\left(k_{e q}-1\right)}\left(\sqrt{\frac{2 z}{\sigma_{s}^{2} \theta_{e q}}}\right) .
\end{aligned}
$$

\section{D. $\alpha$ - $\mu$ Approximation}

In this section, we apply the method of moments to approximate $P_{y_{\text {res }}}$ by an $\alpha-\mu$ distribution. In general, the $\alpha-\mu$ distribution has more degrees of freedom compared to others, since it is capable of representing other distributions. In [13], the sum of i.n.i.d Nakagami RVs has been approximated to an $\alpha-\mu$ distribution. Moreover, $\alpha-\mu$ distribution has been used to approximate the sum of i.n.i.d Gamma RVs [13]. Building upon this, next we describe how to approximate the RV $P_{y_{\text {res }}}$ to an $\alpha-\mu$ distribution. Consequently, the PDF of the RV $P_{y_{\text {res }}}$ can be expressed as follows

$$
f_{P_{y_{r e s}}}(z) \approx \frac{\alpha \mu^{\mu} z^{\alpha \mu-1}}{\hat{r}^{\alpha \mu} \Gamma(\mu)} \exp \left(-\mu \frac{z^{\alpha}}{\hat{r}^{\alpha}}\right),
$$

in which $\hat{r}=\sqrt[\alpha]{E\left[P_{y_{r e s}}^{\alpha}\right]}$ and $\mu=\frac{\hat{r}^{2 \alpha}}{V\left[P_{y_{r e s}}^{\alpha}\right]}$. In order to compute (15), the parameters $\alpha, \mu$, and $\hat{r}$ have to be estimated. The moments estimator for $\alpha$ and $\mu$ are given by [13]

$$
\frac{E^{2}\left[P_{y_{r e s}}\right]}{E\left[P_{y_{r e s}}^{2}\right]-E^{2}\left[P_{y_{r e s}}\right]}=\frac{\Gamma^{2}(\mu+1 / \alpha)}{\Gamma(\mu) \Gamma(\mu+2 / \alpha)-\Gamma^{2}(\mu+1 / \alpha)},
$$

and

$$
\frac{E^{2}\left[P_{y_{r e s}}^{2}\right]}{E\left[P_{y_{r e s}}^{4}\right]-E^{2}\left[P_{y_{r e s}}^{2}\right]}=\frac{\Gamma^{2}(\mu+2 / \alpha)}{\Gamma(\mu) \Gamma(\mu+4 / \alpha)-\Gamma^{2}(\mu+2 / \alpha)} .
$$


In (16) and (17) the moments $E\left[P_{y_{\text {res }}}\right], E\left[P_{y_{r e s}}^{2}\right]$, and $E\left[P_{y_{r e s}}^{4}\right]$, are unknown and are derived next.

Given that $S$ and $H$ are independent RVs, then

$$
E\left[P_{y_{r e s}}^{n}\right]=E\left[S^{n}\right] E\left[H^{n}\right] .
$$

According to (18), the moments of $P_{y_{r e s}}$ can be obtained by computing the moments of $S$ and the moments of $H$. Since $S$ follows a Gamma distribution, its $n$-th moment can be computed by

$$
E\left[S^{n}\right]=((k+n-1)(k+n-2) \cdots k) \times \theta^{n},
$$

where $k=1$ and $\theta=2 \sigma_{s}^{2}$, for the $\mathrm{RX} S$. Lets define $W_{i} \sim$ Nakagami $\left(m_{i}, \lambda_{i}\right)$ as a Nakagami distribution. Then, by definition, $H_{i}=W_{i}^{2}$ for $k_{i}=m_{i}$ and $\theta_{i}=\lambda_{i} / m_{i}$. Therefore, the multi-tap fading channel may be written as $H=\sum_{i=1}^{I} W_{i}^{2}$. Thus, the $n$-th moment of the RV $H$ can be computed using the multinomial expansion as follows

$$
\begin{aligned}
E\left[H^{n}\right] & =\sum_{n_{1}=0}^{n} \sum_{n_{2}=0}^{n_{1}} \cdots \sum_{n_{I-1}=0}^{n_{I-2}}\left(\begin{array}{c}
n \\
n_{1}
\end{array}\right)\left(\begin{array}{l}
n 1 \\
n_{2}
\end{array}\right) \cdots\left(\begin{array}{c}
n_{I-2} \\
n_{I-1}
\end{array}\right) \\
& \times E\left[W_{1}^{2\left(n-n_{1}\right)}\right] E\left[W_{2}^{2\left(n_{1}-n_{2}\right)}\right] \cdots E\left[W_{I}^{2\left(n_{I-1}\right)}\right],
\end{aligned}
$$

where the moments of the RV $W$ are given by

$$
E\left[W_{i}^{n}\right]=\frac{\Gamma\left(k_{i}+n / 2\right)}{\Gamma\left(k_{i}\right)}\left(\theta_{i}\right)^{n / 2} .
$$

Equations (19) and (20) are used to compute the exact moments required in (18). Having obtained $\alpha$ and $\mu$ using (16) and (17), $\hat{r}$ can be estimated by

$$
\hat{r}=\left[\frac{\mu^{2 / \alpha} \Gamma(\mu) E\left[P_{y_{r e s}}\right]}{\Gamma(\mu+2 / \alpha)}\right]^{1 / 2} .
$$

\section{PERFORMANCE ANALYSIS}

In this section we evaluate the accuracy of the two approximations proposed to model the residual SI power. Regarding the simulation, the system design in Fig. 1 is adopted. The carrier frequency is adjusted to $f_{c}=1 \mathrm{GHz}$. The residual SI power is sampled each $\Delta_{T}=1 /\left(360 f_{c}\right)$ s. The values of $X_{r}$ and $X_{j}$ are sampled from Normal distributions each $4 / f_{c}$ with $\sigma_{x}^{2}=\frac{1}{2}$. The values of $Q_{j}^{T, R}$ and $Q_{j}^{T, R}$ are sampled from Normal distributions each $4 / f_{c}$.

The gains of the taps are assumed to be time-variant, so, $H_{r_{i}}$ and $H_{j_{i}}$ are sampled from independent Rician distributions each $40 / f_{c}$. To guarantee a fair comparison, each Rician tap is parameterized with $\mu_{h_{i}}, \sigma_{h_{i}}^{2}$, and $K_{d B_{i}}$ to achieve an average power of $\Omega_{i}=10 \mathrm{~mW}$. Monte Carlo simulations were computed during $277.8 \mu \mathrm{s}\left(10^{8}\right.$ realisations of the stochastic process were run), and the total number of fading taps is set to $I=8$.

The accuracy of the W-S approximation and $\alpha-\mu$ approximation is evaluated for two different scenarios ${ }^{1}$ :

\footnotetext{
${ }^{1}$ The uncertainty refers to the level of dissimilarity of the parameter $K_{d B_{i}}$, and is quantified by the variance of $K_{d B_{i}}$ for the different taps. Regarding Table I and Table II, the index in the first column (" $i / I$ ") represents $i$ for $K_{d B_{i}}, \mu_{h_{i}}, \sigma_{h_{i}}^{2}, \vartheta_{i}^{\circ}, \epsilon_{i}$, and $\phi_{i}^{\circ}$, and represents $I$ for $k_{e q}, \theta_{e q}, \alpha, \mu$, and $\hat{r}$.
}

$\mathrm{S}_{1}$ - in this scenario we consider low uncertainty of the Rician fading taps and the channel estimation errors are relatively small (parameters described in Table I);

$\mathrm{S}_{2}$ - in this scenario we assume that the uncertainty of the Rician fading taps is significantly higher when compared to $\mathrm{S}_{1}$ (parameters in Table II).

TABLE I

FADING TAPS AND ESTIMATION ERRORS ADOPTED IN THE SCENARIO $\mathrm{S}_{1}$.

\begin{tabular}{|c|c|c|c|c|c|c|c|c|c|c|c|}
\hline$" i / I "$ & $K_{d B_{i}}$ & $\mu_{h_{i}}$ & $\sigma_{h_{i}}^{2}$ & $\vartheta_{i}^{\circ}$ & $\epsilon_{i}$ & $\phi_{i}^{\circ}$ & $k_{e q}$ & $\theta_{e q}$ & $\alpha$ & $\mu$ & $\hat{r}$ \\
\hline 1 & -5.0 & 1.55 & 3.80 & 45 & 0.90 & 26 & 1.06 & 1.8 & 1.00 & 1.06 & 0.90 \\
\hline 2 & 0.0 & 2.23 & 2.50 & 60 & 0.80 & 55 & 1.96 & 4.6 & 0.48 & 2.48 & 6.26 \\
\hline 3 & -10.0 & 0.95 & 4.54 & 70 & 0.70 & 10 & 2.40 & 4.3 & 0.48 & 2.72 & 7.21 \\
\hline 4 & -6.0 & 1.41 & 4.00 & 50 & 0.95 & 8 & 2.50 & 4.2 & 0.48 & 2.77 & 7.38 \\
\hline 5 & -9.0 & 1.05 & 4.44 & 30 & 0.75 & 13 & 2.93 & 3.9 & 0.49 & 2.86 & 8.28 \\
\hline 6 & 6.5 & 2.85 & 0.91 & 40 & 0.85 & 24 & 3.78 & 3.5 & 0.50 & 2.89 & 9.80 \\
\hline 7 & 7.4 & 2.91 & 0.77 & 42 & 0.60 & 26 & 5.31 & 3.0 & 0.55 & 2.62 & 12.46 \\
\hline 8 & 8.2 & 2.94 & 0.65 & 46 & 0.80 & 46 & 8.23 & 2.6 & 0.64 & 2.05 & 17.68 \\
\hline $8, \kappa$ & 8.2 & 2.94 & 0.65 & 46 & 0.80 & 46 & 6.50 & 4.0 & 0.61 & 2.17 & 21.50 \\
\hline
\end{tabular}

TABLE II

FADING TAPS AND ESTIMATION ERRORS ADOPTED IN THE SCENARIO $\mathrm{S}_{2}$.

\begin{tabular}{|c|c|c|c|c|c|c|c|c|c|c|c|}
\hline$" i / I "$ & $K_{d B_{i}}$ & $\mu_{h_{i}}$ & $\sigma_{h_{i}}^{2}$ & $\vartheta_{i}^{\circ}$ & $\epsilon_{i}$ & $\phi_{i}^{\circ}$ & $k_{e q}$ & $\theta_{e q}$ & $\alpha$ & $\mu$ & $\hat{r}$ \\
\hline 1 & 10.0 & 3.00 & 0.45 & 60 & 0.70 & 50 & 5.76 & 1.0 & 1.00 & 5.76 & 2.20 \\
\hline 2 & -5.0 & 1.55 & 3.80 & 230 & 0.40 & 200 & 1.78 & 14.0 & 0.43 & 3.10 & 16.44 \\
\hline 3 & 5.0 & 2.75 & 1.20 & 57 & 0.01 & 220 & 3.14 & 11.2 & 0.46 & 3.38 & 25.27 \\
\hline 4 & -10.0 & 0.95 & 4.54 & 32 & 0.6 & 330 & 3.64 & 10.5 & 0.47 & 3.29 & 28.19 \\
\hline 5 & 10.0 & 3.00 & 0.45 & 146 & 0.20 & 146 & 6.21 & 8.4 & 0.56 & 2.63 & 41.29 \\
\hline 6 & -6.0 & 1.40 & 4.00 & 254 & 1.00 & 330 & 6.76 & 8.1 & 0.57 & 2.50 & 43.94 \\
\hline 7 & 50.0 & 0.01 & 5.00 & 310 & 0.87 & 20 & 7.04 & 8.0 & 0.58 & 2.45 & 45.16 \\
\hline 8 & -5.0 & 1.55 & 3.80 & 97 & 0.20 & 85 & 8.07 & 8.2 & 0.63 & 2.10 & 54.83 \\
\hline $8, \kappa$ & -5.0 & 1.55 & 3.80 & 97 & 0.20 & 85 & 6.86 & 11.9 & 0.62 & 2.10 & 67.36 \\
\hline
\end{tabular}

The CDFs of the residual SI power are illustrated in Fig. 2 considering the parameters described in Table I. First, we assume ideal RF and hardware impairments $\left(\kappa_{T}^{2}=\kappa_{R}^{2}=0\right)$ for the simulation of $I=\{2,4,6,8\}$ fading taps. Next, we consider the impact of RF and hardware impairments for $I=8$ taps, with parameters $\kappa_{T}^{2}=\kappa_{R}^{2}=0.1$. The "simulation" curves were obtained through Monte Carlo simulation while "W-S" and " $\alpha$ - $\mu$ " curves were obtained with the computation of the CDFs associated to the PDFs in (14) and (15), respectively. The similarity comparison between simulated data and the proposed approaches indicates a high accuracy of both, $\mathrm{W}-\mathrm{S}$ and $\alpha-\mu$ approximations, when the uncertainty levels of the fading in the different taps is low.

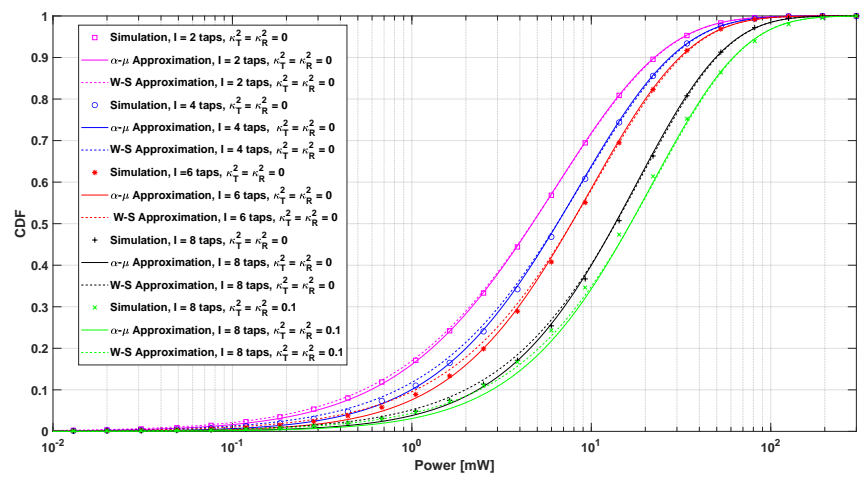

Fig. 2. CDFs of the residual SI power adopting Table I parameters.

The normalized average squared error between the simu- 

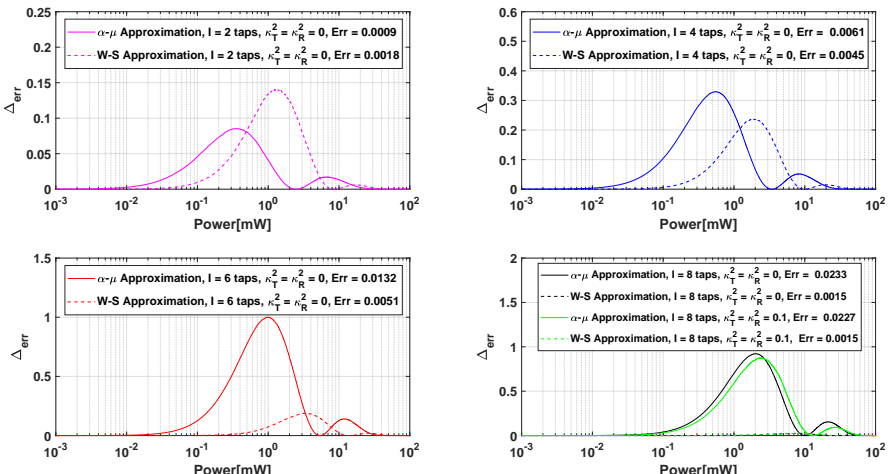

Fig. 3. Normalized square error, $\Delta_{e r r}$ with $\Delta_{\max }=0.242 \times 10^{-3}$, over the power domain for the CDFs plotted in Fig. 2. The average error, Err, is presented in the legend of the figure for each curve.

lated $\left(F_{s}(n)\right)$ and approximated $\left(F_{t}(n)\right)$ CDFs is defined as

$$
E r r=\frac{1}{n_{\max }} \sum_{n=1}^{n_{\max }} \Delta_{e r r}(n),
$$

where $\Delta_{e r r}(n)=\left(F_{s}(n)-F_{t}(n)\right)^{2} / \Delta_{\max }$ is the normalized squared error, $\Delta_{\max }$ is the normalization value, and $n_{\max }$ is the length of the vector containing the non-null squared errors. Fig. 3 plots $\Delta_{e r r}$ values using the CDFs presented in Fig. 2 for each number of fading taps, i.e. considering $I=$ $\{2,4,6,8\}$. The results in Fig. 3 show that the squared error and average error are low, indicating high accuracy of both approaches. However, the W-S approximation exhibits higher levels of accuracy, especially as the number of taps increases. Additionally, the assumption of the impairments increases the power of the residual SI.

Next we evaluate the accuracy of the proposed approximations for the scenario $S_{2}$. Again, (23) is used for the evaluation of the results when utilizing high levels of uncertainty. The simulated and theoretically approximated CDFs are shown in Fig. 4. As can be seen, the W-S approximation exhibits a higher error when compared to the $\alpha-\mu$ approximation, especially for probability values less than 0.5 .

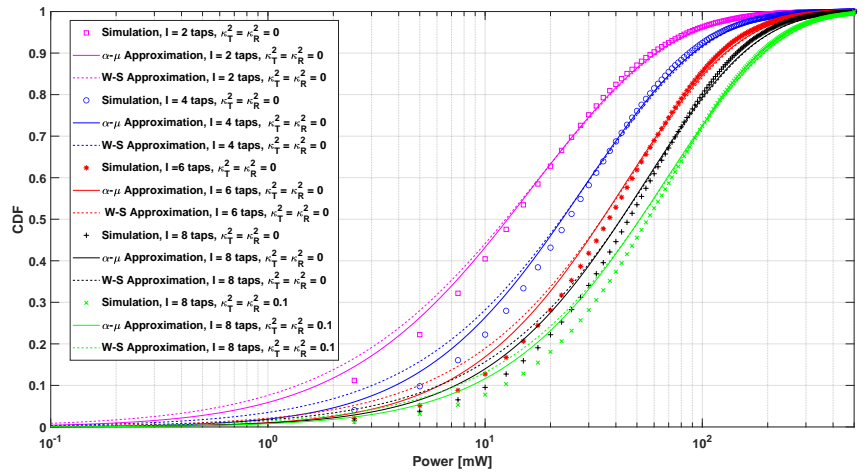

Fig. 4. CDFs of the residual SI power adopting Table II parameters.

Fig. 5 depicts the square error, $\Delta_{e r r}$, values and the average error, $E r r$, values obtained for the $I=\{2,4,6,8\}$ fading taps. The results presented in Fig. 5 indicate that the $\alpha-\mu$ approximation exhibits higher accuracy levels over the W$\mathrm{S}$ approximation when the uncertainty is high. As can be
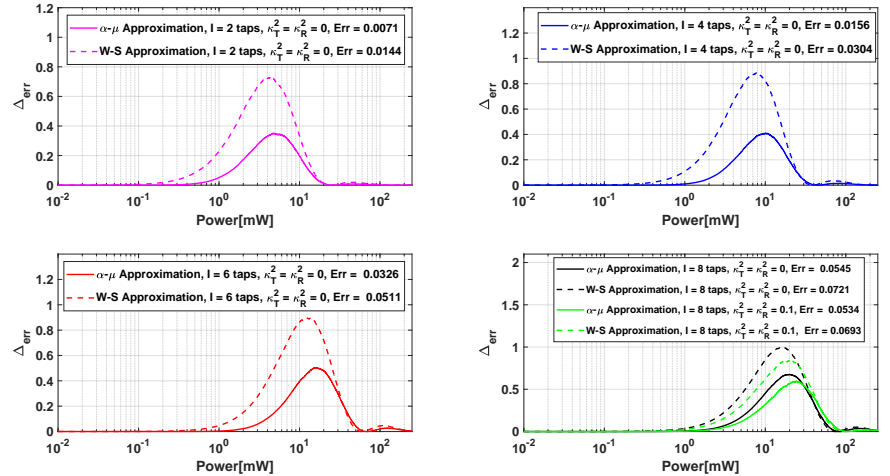

Fig. 5. Normalized square error, $\Delta_{e r r}$ with $\Delta_{\max }=4.6 \times 10^{-3}$, over the power domain for the CDFs plotted in Fig. 4. The average error, Err, is presented in the legend of the figure for each curve.

seen, the accuracy of both approximations decreases with the number of taps. Error values are also reported when considering RF and hardware impairments in both scenarios, $\mathrm{S}_{1}$ and $\mathrm{S}_{2}$, for $I=8$ taps.

\section{CONCLUSIONS}

This work derives closed-form approximations for the distribution of the residual SI power caused by multi-tap delay fading channels in IBFDX systems. Through the comparison of numerical and simulated results, we have shown that the residual SI power can be accurately approximated using the $\mathrm{W}-\mathrm{S}$ equation when adopting low levels of fading uncertainty, although the $\alpha-\mu$ moment-based approximation exhibits higher accuracy when the uncertainty increases.

\section{REFERENCES}

[1] K. E. Kolodziej, B. T. Perry, and J. S. Herd, "In-band full-duplex technology: Techniques and systems survey," IEEE Trans. Microw. Theory Techn., vol. 67, no. 7, pp. 3025-3041, July 2019.

[2] G. C. Alexandropoulos and M. Duarte, "Joint design of multi-tap analog cancellation and digital beamforming for reduced complexity full duplex MIMO systems," in IEEE Int. Conf. on Communications, 2017, pp. 1-7.

[3] A. Sahai, G. Patel, C. Dick, and A. Sabharwal, "On the impact of phase noise on active cancelation in wireless full-duplex," IEEE Trans. Veh. Technol., vol. 62, no. 9, pp. 4494-4510, Nov 2013.

[4] A. Mohammadian and C. Tellambura and M. Valkama, "Analysis of SelfInterference Cancellation Under Phase Noise, CFO, and IQ Imbalance in GFDM Full-Duplex Transceivers," IEEE Trans. Veh. Technol., vol. 69, no. 1, pp. 700-713, 2020.

[5] A. Masmoudi and T. Le-Ngoc, "A Maximum-Likelihood Channel Estimator for Self-Interference Cancelation in Full-Duplex Systems," IEEE Trans. Veh. Technol., vol. 65, no. 7, pp. 5122-5132, 2016.

[6] L. Irio and R. Oliveira, "On the impact of fading on residual selfinterference power of in-band full-duplex wireless systems," in IWCMC, 2018, pp. 142-146.

[7] L. Irio and R. Oliveira, "Distribution of the residual self-interference power in in-band full-duplex wireless systems," IEEE Access, vol. 7, pp. 57 516-57 526, 2019.

[8] E. Bjornson and M. Matthaiou and M. Debbah, "A New Look at DualHop Relaying: Performance Limits with Hardware Impairments," IEEE Trans. Commun., vol. 61, no. 11, pp. 4512-4525, 2013.

[9] B. C. Nguyen, X. N. Tran, D. T. Tran, X. N. Pham, and L. T. Dung, "Impact of hardware impairments on the outage probability and ergodic capacity of one-way and two-way full-duplex relaying systems," IEEE Trans. Veh. Technol., vol. 69, no. 8, pp. 8555-8567, 2020.

[10] Y. Choi and H. Shirani-Mehr, "Simultaneous Transmission and Reception: Algorithm, Design and System Level Performance," IEEE Trans. Wireless Commun., vol. 12, no. 12, pp. 5992-6010, 2013.

[11] F. E. Satterthwaite, "An approximate distribution of estimates of variance components," Biometrics Bulletin, vol. 2, no. 6, pp. 110-114, 1946. 
[12] B. L. Welch, "The generalization of 'student's' problem when several different population variances are involved," Biometrika, vol. 34, no. 1/2, pp. 28-35, 1947.

[13] D. B. da Costa, M. D. Yacoub, and J. C. S. Santos Filho, "An improved closed-form approximation to the sum of arbitrary nakagami$m$ variates," IEEE Trans. Veh. Technol., vol. 57, no. 6, pp. 3854-3858, Nov 2008. 